\title{
DIVERSIDAD DE FAUNA SILVESTRE EN BOSQUE SECO TROPICAL, NANDAROLA, NANDAIME
}

\author{
Miguel Garmendia-Zapata ${ }^{1}$, Carlos Lenin Tercero-Sánchez ${ }^{2}$ \\ ${ }^{1}$ Docente de la Facultad de Recursos Naturales y del Ambiente, UNA, Managua, Nicaragua. \\ ${ }^{2}$ Egresado de la Carrera de Ingeniería en Recursos Naturales Renovables, FARENA/UNA
}

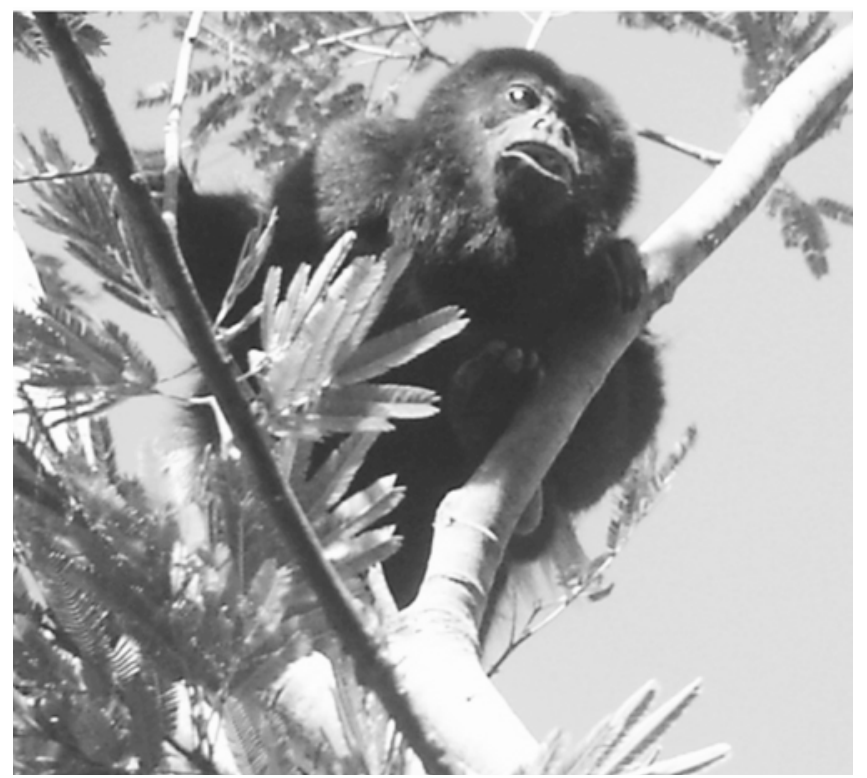

\section{RESUMEN}

Frente a los efectos de la degradación de hábitat por fragmentación que enfrentan los bosques de nuestro país, es importante conocer las especies de fauna silvestre que mantienen relación ecológica con estos fragmentos aislados y que promueven el desarrollo de la vegetación a consecuencia de sus actividades y hábitos. El objetivo de este estudio es determinar y comparar la diversidad de fauna silvestre (vertebrados terrestres) en dos fragmentos de bosque seco secundarios de diferentes edades y tamaños (15 años, 3.5 ha; 11 años, 2.8 ha) en bosque seco tropical, Nandarola, Nandaime, e identificar los gremios alimenticios de las especies. Se establecieron seis transeptos de $20 \times 100 \mathrm{~m}$ en el fragmento de 15 años denominado "La Chipopa” y tres transeptos de iguales dimensiones en el fragmento de 11 años denominado “La Zorra”, en estos se cuantificó la diversidad de anfibios, reptiles y mamíferos. En el extremo de cada transepto se estableció un punto de muestreo de $25 \mathrm{~m}$ de radio para evaluar diversidad de aves, se realizaron 17 observaciones distribuidas en ocho meses. Como resultados se determinaron 2 especies de anfibios, 10 de reptiles, 26 de aves y 7 de mamíferos. La riqueza de especie para los cuatro grupos fue mayor en La Zorra (69 especies) comparada con La Chipopa (49 especies). Los índices demostraron una mayor diversidad de reptiles, aves y mamíferos en La Zorra al compararse con La Chipopa según los índices ecológicos de Shannon-Wiener y Simpson. Se identificaron nueve gremios alimenticios en los
ABSTRACT

Considering the effects of habitat degradation due to forest fragmentation that Nicaragua is facing, it is extremely important to know wildlife species that maintain ecological relationship and promote the development of vegetation as a result of their activities and habits. The goal of this study was to determine and compare the diversity of wildlife (terrestrials vertebrates) into two fragments of dry forest with different ages and sizes (15 years old, $3.5 \mathrm{ha}, 11$ years old, $2.8 \mathrm{ha}$ ) and identify the food guild of species found. Six transects 20 x 100 m were placed in the fragment forest aged 15 years (La Chipopa), and three transects with the same size in the fragment forest aged 11 years (La Zorra). The diversity of amphibians, reptiles and mammals species were evaluated. At the end of each transept a sampling point was established, it had a radio of $25 \mathrm{~m}$ in order to assess birds Diversity. There were 17 observations spread over eight months. As a results 2 species of amphibians were determined, 10 reptiles, 26 birds and 7 mammals. Species richness for the four groups was higher in La Zorra (69 species) compared with La Chipopa (49 species). The diversity rates Shannon-Wiener and Simpson, showed greater diversity of reptiles, birds and mammals at La Zorra. Nine food associations were identified in the two forests fragments. Insectivorous was the largest food association, involving 26 species. Considering food guilds that generate seeds and fruits dispersals and work in pollination, Frugivorous, Seeds Eater and Nectarivoros, were determined 12, 2 and 10 species respectively. Despite the differences in ages and sizes, La Zorra had more wildlife diversity compared 
involucrando a 26 especies, para los gremios importantes en la dispersión de frutos y semillas, y en la polinización: los Frugívoros, Granívoros Nectarívoros fueron determinadas 12, 10 y 2 especies respectivamente. Pese a las diferencias en edades y tamaños el fragmento de La Zorra presenta la mayor diversidad de vida silvestre comparado con La Chipopa, pero esas diferencias no son significativas. En sí la presencia de dispersores de semillas y frutos es muy baja en ambos sitios, en cuanto a los vertebrados polinizadores solo se determinó una especie. El 44\% de las especies son exclusivas de bosques intervenidos y lugares abiertos.

Palabras Clave: Fragmentación, Gremios Alimenticios, Vertebrados Terrestres. with La Chipopa, but these differences were not significant. The presence of Frugivorous and seeds eaters was very low in both places. There was one pollinator specie. The $44 \%$ of the species were exclusively from managed forests and opened areas.

Keywords: Fragmentation, Food Association, Terrestrials Vertebrates
$\mathrm{N}$ icaragua tiene 250,000 ha de bosque seco, lo que representa un $2 \%$ de la cobertura forestal (Harcourt and Sayer, 1996). De acuerdo con Roldan (2001), el 80\% ha sido convertido a tierras agrícolas y ganaderas. Uno de los factores causante de extinciones locales de especies de fauna silvestre además de la captura comercial, el tráfico ilegal y la cacería es la destrucción y degradación de sus hábitats (Hurtado, 2000).

Antes de definir y entender con más detalle la influencia de la fauna silvestre en un bosque (relación planta-animal), es necesario en primer lugar cuantificar la diversidad de especies de animales dentro del bosque, sus hábitos, hábitat, comportamiento y alimentación, conociendo esta información se pueden identificar las especies que tienen un influencia directa y potencialmente en el desarrollo y mantenimiento de la estructura y diversidad del bosque y dirigir investigaciones hacia estas(Guariguata y Kattan, 2002).

Existe poca información a nivel nacional sobre el monitoreo de los cambios en la abundancia y diversidad de la fauna silvestre en la sucesión de los bosque, principalmente bosques secos.

En este estudio nos proponemos determinar la abundancia y diversidad de fauna silvestre específicamente de los animales vertebrados terrestres de las clases Amphibia, Reptilia, Aves y Mammalia de dos fragmentos de bosque los cuales fueron áreas agrícolas abandonadas con diferentes edades y áreas, y a la vez, comparar dicha abundancia y diversidad entre ellos. Además se pretende conocer la diversidad de gremios alimenticios a los cuales están asociadas las especies de fauna silvestre, el estado de conservación a los que pertenece cada especie y las especies incluidas dentro del protocolo de la convención sobre el Comercio Internacional de Especies Amenazadas de Fauna y Flora Silvestres (CITES).

\section{MATERIALES Y MÉTODO}

Este trabajo está basado principalmente en la detección e identificación de animales silvestres de las clases Mamíferos, Aves, Reptiles y Anfibios, mediante el método de avistamiento dentro de las unidades de muestreo, con la ayuda de Binoculares 7x35 y 10x50. La información de campo fue enriquecida con revisión de bibliográfica, utilizando libros reconocidos a nivel del Neotrópico.

Descripción del sitio. El sitio de estudio se localiza en el bosque seco de Nandarola a 7-10 Km de la Ciudad de

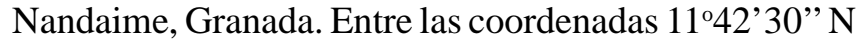
y $86^{\circ} 05^{\prime} 30^{\prime \prime} \mathrm{W}$. El clima predominante es típico de las zonas cálidas secas y la temperatura oscila entre $26^{\circ} \mathrm{C}$ y $29^{\circ} \mathrm{C}$, con una precipitación media anual entre 900 y 1300 mm, la época lluviosa es de Mayo a Octubre. Las condiciones topográficas dentro del bosque estudiado las podemos clasificar en tres tipos: áreas planas, áreas onduladas, áreas quebradas y en algunos casos escarpados (MARENA, 1994). La diferencia de elevación dentro del área esta entre 211 msnm, (parte plana) y 261 msnm, (en la parte quebrada) predominando el relieve ondulada con pendientes del 30\% aunque existen entre $45 \%$ y $80 \%$ en trechos cortos (MARENA, 1994).

\section{PROCESO METODOLÓGICO}

Unidades de muestreo. Para la cuantificación de los vertebrados terrestres, se utilizaron dos tipos de unidades de muestreo: transeptos de ancho fijo 20x100m para realizar el muestreo de Mamíferos, Reptiles y Anfibios, y puntos de muestreo de $25 \mathrm{~m}$ de radio para evaluar al grupo de las Aves. Para efecto de una mejor comodidad en campo y optimizar el tiempo, la unidades redondas se ubicaron en uno de los extremos de las unidades rectangulares, conformando una sola unidad (Figura 1). 


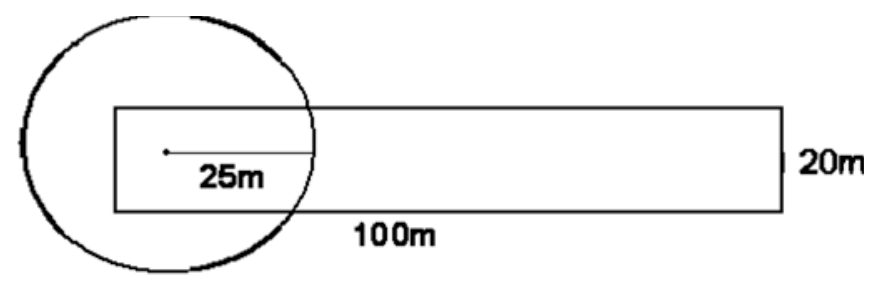

Figura 1. Transeptos de ancho fijo y puntos de muestreo formando una sola unidad.

Diseño de muestreo. El estudio se delimitó a dos áreas de cultivo abandonadas, uno localizado en la comunidad "La Chipopa" (15 años, 3.5 ha) y otro en la comunidad "La Zorra" (11 años, 2.8 ha). Los conjuntos transepto-puntos se colocaron sistemáticamente a lo largo de las dos áreas, separados aproximadamente 100 $\mathrm{m}$ uno de otro. El extremo de cada transepto en el que se colocó la parcela circular se hizo al azar. En la Chipopa se colocaron seis unidades muéstrales y en la Zorra se colocaron tres (Figura 2, A y B). La diferencia entre los

A

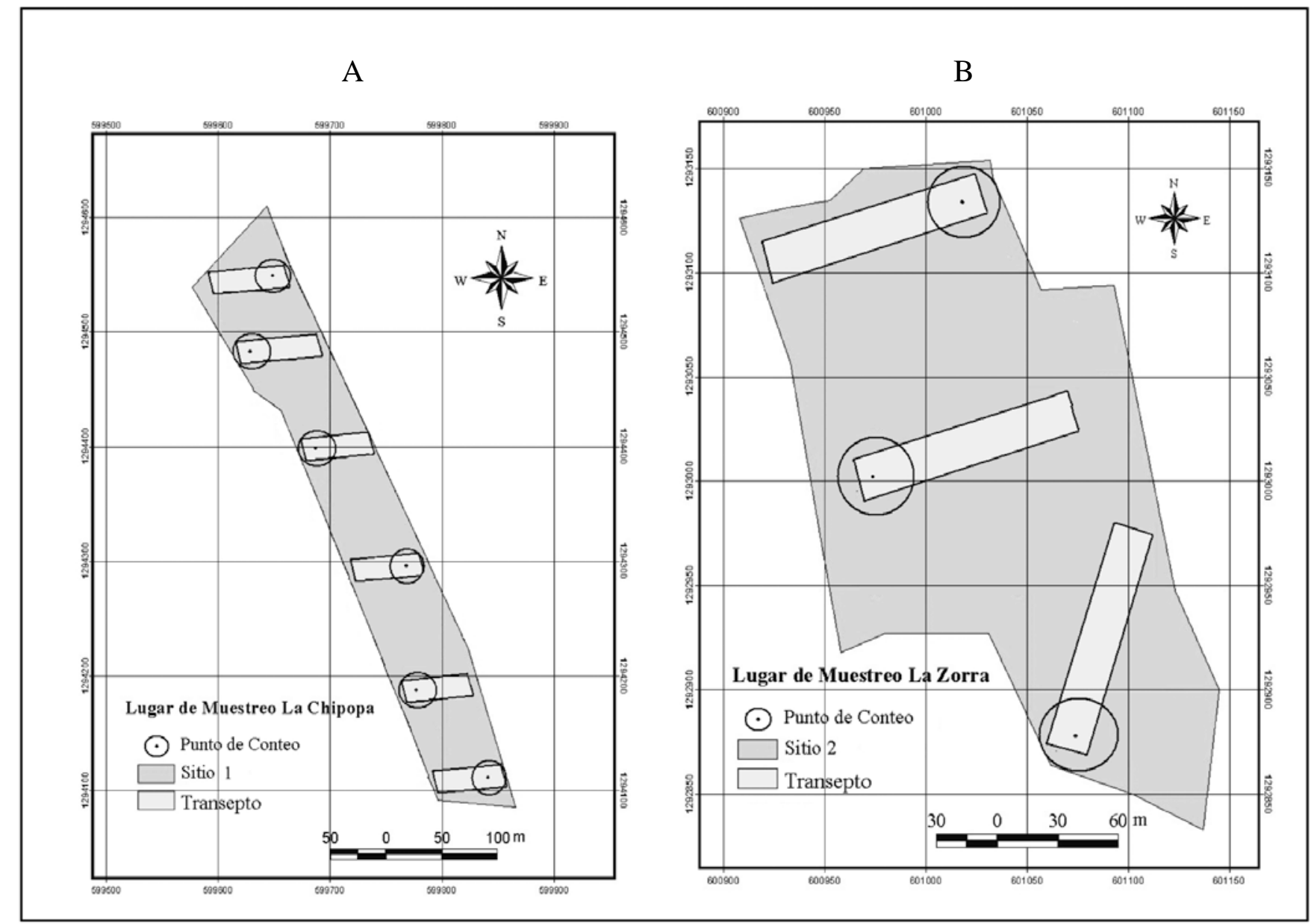

números de unidades de muestro en cada sitio se debe exclusivamente a la forma y tamaño de éstos.

Se realizaron 17 observaciones a lo largo de ocho meses (octubre-mayo), cada observación corresponde a un día en el cual se muestreo por la mañana (07:0011:00 am), la noche (06:00-10:00 pm) y en la madrugada (05:00-07:00 am). El muestreo en los transeptos se llevo a cabore corriendo estos de extremo a extremo, a una velocidad promedio de un kilómetro por hora, sigilosamente, revisando los estratos arbustivos y arbóreos, buscando bajo troncos caídos y bajo piedras. En cuanto a los puntos de muestreo, estos se revisaron a la misma hora en que se revisaban los transeptos, las observaciones en estos se llevaban a cabopor 10 minutos, en ese tiempo, se identificaban y se contaban las aves directamente observándolas o indirectamente por sus cantos.

Variables a medir. Para la toma de datos se requirió de un formulario de campo sencillo que facilitó el levantamiento de la información. Las variables medidas

Figura 2. Diseño de muestro (A) seis conjuntos transepto-punto colocados a lo largo del parche abandonado de la Chipopa, (B) tres conjunto transepto-punto colocados en el parche abandonado de la Zorra. 
fueron: Número de Individuos; Número de especies, Alimento. La identificación se llevó a cabo con el apoyo de guías de campo ilustradas para vertebrados terrestres como lo son: Neotropical Reinforest Mammals (Emmons, 1999); Mamíferos Silvestres de la Cuenca de México (Ceballos y Galindo, 1984); Bird of Costa Rica (Stiles \& Skutch, 2007); Guía Ilustrada de Herpetofauna de Nicaragua (Ruiz y Buitrago, 2003); Reptiles de Centro América (Kohler, 2003). En el caso del alimento se observó a la medida de lo posible de que se estaba alimentando cada animal en el momento de ser avistado. Los datos de esta variable fueron complementados con revisión bibliográfica, ya que en la mayoría de los casos fue difícil lograr observar a los animales alimentándose.

También se determinó el estado de conservación del ecosistema a los que pertenece cada especie: se revisó bibliografía para conocer el estado de conservación del ecosistema en el que son frecuentes encontrar cada una de las especies, esto es importante para identificar especies indicadoras de sitios alterados o de sitios conservados. Las especies se determinaron al revisarse las listas de los apéndices I, II y III de las normas y procedimientos para la exportación e importación de especies de flora y fauna silvestre de Nicaragua.

Análisis de la información. Las variables medidas en campo y los datos de origen bibliográficos fueron utilizadas en el cálculo de la Densidad o número de individuos por unidad de área, Riqueza de Especie que consiste solamente en un conteo de las especies. Para determinar la diversidad estructural se calcularon los índices de diversidad de Shannon-Wiener y Simpson. Para el primero se utilizó la fórmula $\mathrm{H}^{\prime}=-\sum[(\mathrm{ni} / \mathrm{n}) \ln$ $(\mathrm{ni} / \mathrm{n})]$ y para el índice de Simpson se utilizó la fórmula $\lambda$ $=\sum \mathrm{ni}(\mathrm{ni}-1) / \mathrm{n}(\mathrm{n}-1)$ donde, $\mathrm{ni}=$ Número de individuos pertenecientes a la $\mathrm{i}$ - ésima especie de la muestra.

Con los datos de alimento se determinaron los gremios alimenticios. Las especies se agruparon también en base al estado de conservación del ecosistema a los que pertenece cada una, esto se llevó a cabo a través de revisión bibliográfica. Previamente se clasificaron los tipos de ecosistemas en base a la descripción de la historia natural de cada animal en las literaturas antes mencionadas como: bosque no intervenido; bosque poco intervenido; bosque intervenido lugares abiertos y áreas urbanas sub-urbanas.

\section{RESULTADOS Y DISCUSIÓN}

Descripción general de la fauna silvestre. Se Contaron 118 individuos los cuales están agrupados en 33 familias, 45 géneros y 45 especies de fauna silvestre. De estas especies 2 son anfibios, 10 reptiles, 26 aves y siete mamíferos.

Diversidad de aves. Fueron observados 86 individuos aves agrupados en 20 familias, 26 géneros y 26 especies. La abundancia y la riqueza fue mayor en La Zorra comparada con La Chipopa, dichas diferencias resultaron no ser significativas (abundancia: $\mathrm{U}_{35 \mathrm{gl}}=161, \mathrm{p}>0.84$; riqueza: $\mathrm{U}_{35 \mathrm{gl}}=148.5$, $\mathrm{p}>0.56$ ).

Las especies más abundantes en ambos sitios fueron Campylorynchus rufinucha, Eumomota supersiliosa, Piaya cayana, Thryothorus pleurostictus representadas por 5-10 individuos, el resto estaba representado por menos de 4 individuos. La Zorra presentó una mayor diversidad biológica de aves según los índices de Simpson y Shannon-Wiener, estas diferencias solo resultaron ser significativas para el índice de Shannon-Wiener (Simpson: $\mathrm{U}_{7 \mathrm{gl}}=2, \mathrm{p}>0.095$; Shannon-Wiener: $\mathrm{T}_{7 \mathrm{gl}}=$ 2.404, $\mathrm{p}<0.047$ ), (Tabla 1).

Esto nos demuestra que aunque la riqueza y la abundancia son iguales, cuando se emplea un índice para relacionar las dos variables y determinar la equidad en la distribución del número de individuos por especies, resulta que La Zorra probablemente tenga una distribución individuos-especies más equitativa.

Diversidad de mamíferos. Se observaron 15 individuos agrupados en 6 familias, 7 géneros y 7 especies de mamíferos. La abundancia y la riqueza fue mayor en $\mathrm{La}$ Zorra, dichas diferencias resultaron no ser significativas (abundancia: $\mathrm{T}_{6 \mathrm{gl}}=1.14, \mathrm{p}>0.30$; riqueza: $\mathrm{T}_{6 \mathrm{gl}}=1.035$, $\mathrm{P}>0.34)$.

Las especies más abundantes en ambos sitios fueron Sciurus variegatoides y Didelphis virginianus representadas por 6 y 4 individuos respectivamente, el resto estaba representado por 2 ó 1 individuo. La Zorra presentó una mayor diversidad biológica de mamíferos según los índices de Simpson y Shannon-Wiener, (Tabla 1 ), aunque no se aplicó estadística para explorar relación entre los dos grupos por falta de información.

Los dos sitios son iguales en abundancia, riqueza y presuntamente en diversidad, predominando especies de hábitat abierto y que tienen alta relación con las actividades humanas (S. variegatoides y P. opossum).

Diversidad de reptiles. Se observaron?18 individuos agrupados en 5 familias, 10 géneros y 10 especies de reptiles. La abundancia fue mayor en la Chipopa y la riqueza fue igual para ambos sitios, las diferencias para la abundancia resultaron no ser significativas (abundancia: $\mathrm{T}_{7 \mathrm{gl}}=$ 1.805, $\mathrm{P}>0.11)$. 
La especie más abundante en ambos sitios fueron Ameiva quadrilineata con 12 individuos, seguido de Rhinochemmys pulcherrima y Sceleoporus squamosus representadas 4 individuos cada una, el resto estaba representado por 2 ó 1 individuo. La Zorra presentó una mayor diversidad biológica de reptiles según los índices de Simpson y Shannon-Wiener, (Tabla 1), aunque no se aplicó estadística para explorar relación entre los dos grupos por falta valores suficientes.

La riqueza y la abundancia no tienen diferencias significativas al comparar al grupo de los reptiles entre los dos sitios, pese a que solo se comparten $20 \%$ de las especies, es muy probable que con un mayor esfuerzo de muestreo se determine la misma composición de especies para cada sitio.

Diversidad de anfibios. Solamente se encontraron dos especies de anfibios cada una representada por un individuo, dichas especies son: Bufo marinus de la familia BUFONIDAE en La Chipopa y Physalaemus pustulosus de la familia LEPTODACTYLIDAE en La Zorra. No se puede afirmar que estas dos especies sean poco abundantes en los dos sitios, ya que en el estudio solo se cuantificaron los animales que eran encontrado dentro de los transeptos, desafortunadamente en los transeptos no fueron localizado suficientes charcas, ni cruzaban cuerpos de agua que propiciasen las condiciones para observar anfibios.

En general no hay diferencias significativas en cuanto a diversidad de fauna silvestre entre los dos fragmentos de bosque, lo que evidencia que sus diferencias de edades y de tamaño no son suficientes para que dicha diversidad difiera en ambos sitios. Sin embargo es notorio que La Zorra tiene la mayor cantidad de especies y de individuos, varias hipótesis pueden surgir del por que La Zorra siendo el bosque más joven es más rico y abundante, en primer lugar es posible argumentar que dentro de este existían mosaicos de hábitats, tales como tacotales, charrales bajos, pastizales y un cultivo en el centro del área forestal, esto es un factor importante en la diversificación del hábitat, ya que la diversidad de aves obedece más a la diversidad estructural de las plantas que a la diversidad de especies (Krebs, 1985), por otra parte en La Zorra fueron observados mayor cantidad de árboles remanentes y de gran porte comparado con La Chipopa.

Gremios alimenticios. Para las cuatro clases estudiadas fueron determinados nueve gremios alimenticios los cuales son Carnívoros, Insectívoros, animales que se alimentan de invertebrados no insectos, Carroñeros,
Herbívoros, Frugívoros, Granívoros, Nectarívoros y Omnívoros.

A como muestra la Figura 3 los gremios de los invertebrados (insectívoros y consumidores de invertebrados no insectos) son los que tiene más especies involucradas (32 especies). Las especies con funciones ecológicas de dispersión de frutos y semillas perteneciente a los gremios Frugívoros y Granívoros involucran a relativamente pocas especies (12 y 10 especies respectivamente).

Sin embargo los gremios no son específicos de cada especie, una especie puede estar involucrada en más de dos gremios, y así, tener poca especificidad de alimentación. Esta investigación tiene un interés enfatizado en la determinación de especies potenciales como frugívoras y granívoras, especies cuya actividad dispersoras influye en el éxito reproductivo y estructura de las comunidades vegetales (Guariguata y Kattan, 2002). La Tabla 2, enlista las especies frugívoras y granívoras determinadas en los dos sitios de estudio, cabemencionar que la mayoría de estas especies están involucradas en uno o dos gremios, únicamente $C$. inca es una especie estrictamente granívora, en cuyo caso dispersa semillas de tamaño pequeño principalmente de plantas herbáceas principalmente de Gramíneas y Solanáceas, entre otras, también en literatura se conoce que ingiere areniscas del suelo.

Entre las especies con potenciales para dispersar semillas y frutos grandes de árboles como los de las familias Moraceae, Euphorbiaceae, Fabaceae, Caesalpiniaceae, Mimosaceae, Meliaceae, etc. están en su mayoría los mamíferos, entre los que podemos mencionar A. palliata, $S$. variegatoides, las aves como $B$. jugularis, y algunos de menor potencial como M. hofmannii y P. flavus.

Es notoria la ausencia de otros animales que son potencialmente dispersores como lo son algunos mamíferos de tamaño mediano miembros de los Ordenes Rodentia (Agouti, Dasyprocta), Artiodactyla como los de la Familia Tayassuidae, y algunos miembros del Orden Carnívora que complementan su dieta con frutos como los de la Familia Procyonidae, también entre las aves el grupo de los Psittacidos (excepto $B$. jugularis) y Ramphastos entre otros. Muchos estudios han demostrado que si se excluyera a los mamíferos (por ejemplo), la supervivencia y el crecimiento de las plántulas de muchas especies mejoraría notablemente aunque la diversidad de especies de la comunidad podría disminuir (Guariguata y Kattan, 2002), por efecto de monopolización del recurso.

Estado de conservación de los hábitats en las que son comunes encontrar cada especie. Nos referimos a los estados de conservación en los que se encuentran 
Tabla 1. Índices de diversidad de aves, mamíferos y reptiles para ambas localidades

\begin{tabular}{lllllll}
\hline & Aves & \multicolumn{3}{c}{ Mamiferos } & \multicolumn{2}{c}{ Reptiles } \\
\hline Indice de diversidad & La Chipota & La Zorra & La Chipota & La Zorra & La Chipota & La Zorra \\
& & & & & & \\
\hline Simpson & 0.23 & 0.09 & 0.50 & 0.35 & 0.47 & 0.44 \\
Shannon-Wiener & 1.62 & 2.50 & 0.69 & 1.12 & 0.82 & 0.91 \\
& & & & & & \\
\hline
\end{tabular}

los hábitats en los cuales son frecuentes encontrar cada especie. Este es un dato muy importante desde la perspectiva de encontrar especies indicadoras de áreas degradadas o determinar la remanencia de especies del bosque original. En este sentido en nuestro estudio determinamos que las 45 especies de fauna silvestre de los dos sitios estudiados pertenecen a hábitat de bosques intervenidos y lugares abiertos, y 16 son animales frecuentes en áreas Urbanas o Suburbanas. De igual forma, seis especies pertenecen a hábitat de bosques no intervenidos y 14 a hábitats de bosque poco intervenidos.

Las especies que pertenecen a un único estado de conservación de hábitat, resulto que son exclusivas de bosques intervenidos y lugares abiertos. Esto es evidencia de que la fauna silvestre presente en los dos sitios es típica de esos tipos de hábitat (bosques intervenidos y lugares abiertos) y que son especies que estarán formando parte de los bosques jóvenes hasta que la estructura, composición y diversidad de la vegetación, cambien gracias a la sucesión natural. A largo plazo sería de interés el continuar desarrollando investigaciones en los mismos sitios para determinar cambios en la abundancia, riqueza y diversidad de fauna silvestre a la par de los cambios que sufran las comunidades vegetales.
Especies CITES. Fueron determinadas seis especies CITES (Convención sobre el Comercio Internacional de Especies Amenazadas de Fauna y Flora Silvestres) pertenecientes al apéndice II y III (Tabla 3), estas se encuentran repartidas equitativamente (tres para La Chipopa y tres para La Zorra) entre las dos áreas de estudio. Las especies pertenecientes a estos dos apéndices no están necesariamente en peligro de extinción pero su comercio es reglamentado para evitar ese estado (Montenegro, 2007).

Las densidades en las que se encontraron las especies son relativamente bajas, solo $A$. cyanura y $O$. cooperi se encuentran en densidades de cinco individuos por hectárea ambos en el sitio de La Zorra. Cuatro de las seis especies CITES son de Bosques Intervenidos y Lugares Abiertos, las restantes dos se encuentran en casi todos los tipos de estado de conservación de hábitat.

\section{CONCLUSIONES}

En las áreas de cultivo abandonadas de las comunidades La Chipopa y La Zorra fueron determinados 45 especies de fauna silvestre, agrupadas en 45 géneros y 32 familias. De estas especies dos son Anfibios, 10 Reptiles, 26 Aves y siete Mamíferos.

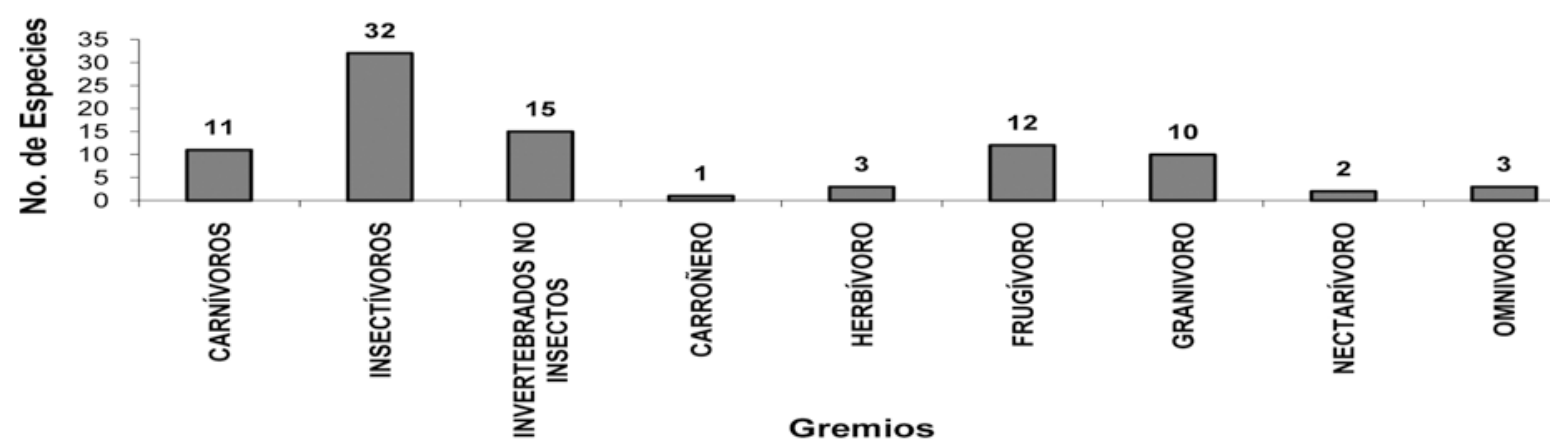

Figura 3. Gremios alimenticios en los que se agrupan las especies de fauna silvestre determinada en el campo. 
Las especies más abundantes fueron: Campylorynchus rufinucha, Eumomota supersiliosa, Piaya cayana, Thryothorus pleurostictus (Aves); Sciurus variegatoides y Didelphis virginianus (Mamíferos); Ameiva quadrilineata, Rhinochemmys pulcherrima y Sceleoporus squamosus (Reptiles); para Anfibio fueron determinadas solamente 2 especies Bufo marinus y Physalaemus pustulosus.

Al comparar la abundancia, riqueza y diversidad de vertebrados terrestres entre los dos sitios no se determinaron diferencias significativas para las dos primeras variables y los índices de diversidad solo fueron significativos para Shannon-Wiener en Aves siendo La Zorra más diverso, en cuanto a la diversidad de mamíferos y reptiles, La Zorra presentó mayor
Las 45 especies de fauna silvestre pertenecen a un sitio intervenido o lugares abiertos, algunas de estas también pueden ser frecuentemente encontradas en bosque no intervenidos (6 spp), poco intervenidos (14 spp) y áreas urbanas (15 spp).

Las especies CITES determinadas fueron Brotogeris juguaris (Apéndice II), Amazilia cyanura (Apéndice II), Iguana iguana (Apéndice II), Otus cooperi (Apéndice II), Potos flavus (Apéndice III) y Crotalus durissus (Apéndice III).

\section{RECOMENDACIONES}

Disminuir presión sobre fauna silvestre principalmente sobre las especies de mamíferos de tamaño mediano y grande.

Tabla 2. Lista de especies Frugívoras y Granívoras y la cantidad de gremios a los que pertenecen, para los dos sitios de estudio

\begin{tabular}{lccc}
\hline Especies & Frugívoros & Granívoros & $\begin{array}{c}\text { Cantidad de gremios a } \\
\text { los que pertenece }\end{array}$ \\
\hline $\begin{array}{l}\text { Aimophila ruficauda } \\
\text { Alouatta palliata } \\
\text { Brotogeris juguaris }\end{array}$ & $\mathrm{X}$ & $\mathrm{X}$ & 3 \\
Colinus leucopogon & $\mathrm{X}$ & $\mathrm{X}$ & 2 \\
Columbina inca & $\mathrm{X}$ & $\mathrm{X}$ & 2 \\
Ctenosaura similis & $\mathrm{X}$ & $\mathrm{X}$ & 3 \\
Icterus pustulatus & $\mathrm{X}$ & $\mathrm{X}$ & 1 \\
Leptotila verreavii & & $\mathrm{X}$ & 2 \\
Melanerpes holfmannii & $\mathrm{X}$ & & 4 \\
Myiarchus tyrannulus & & $\mathrm{X}$ & 2 \\
Pachyrampuhus aglaiae & $\mathrm{X}$ & $\mathrm{X}$ & 2 \\
$\begin{array}{l}\text { Pitangus sulphuratus } \\
\text { Potos flavus }\end{array}$ & $\mathrm{X}$ & & 2 \\
Sciurus variegatoides & $\mathrm{X}$ & $\mathrm{X}$ & 3 \\
Trogon melanocephalus & $\mathrm{X}$ & $\mathrm{X}$ & 3 \\
Turdus grayi & $\mathrm{X}$ & & 2 \\
& $\mathrm{X}$ & & 3 \\
\hline
\end{tabular}

diversidad de mamíferos y la Chipopa mayor de reptiles. En cuanto a anfibios no se hicieron las comparaciones porque sólo se encontraron dos especies una en cada sitio.

Se determinaron 9 categorías en cuanto a gremios alimenticios, entre ellas: los carnívoros, insectívoros, consumidores de invertebrados no insectos, carroñeros, herbívoros, frugívoros, granívoros, nectarívoros y omnívoros, de los cuales los más abundantes en cuanto a número de especies involucradas fueron los insectívoros siendo las aves las principales consumidoras de insectos.
Reforestar y enriquecer con especies de árboles nativos que sean abundantes en frutos y semillas que sirvan de alimento y hábitat a la fauna silvestre como lo son: Acetuno (Simarouba glauca), Aromo (Acasia farmesiana), Ceiba (Ceiba pentandra), Coyol (Acrocomia vinifera), Cuajiniquil (Inga vera ssp. Spuria), Genízaro (Pithecelobium saman), Guacimo de ternero (Guazuma ulmifolia), Guanacaste negro (Enterolobium cyclocarpun), Jaboncillo (Sapindus saponaria), Jocote garrobero (Spondias purpurea), Jocote Jobo (Spondias mombin), Mora (Chlorophora tinctoria), Muñeco (Cordia collococa), Tigüilote 
Tabla 3. Clasificación de las especies encontradas dentro de la CITES

\begin{tabular}{|c|c|c|}
\hline Especies & Apéndices & $\begin{array}{l}\text { Estado de conservación del hábitat } \\
\text { Al que pertenecen las especies }\end{array}$ \\
\hline
\end{tabular}

Densidad en el área de estudio Individuos por ha

\begin{tabular}{|c|c|c|c|c|}
\hline & & & La Chipota & La Zorra \\
\hline Amazilia cyanura & II & B. intervenido/ lugares abiertos & 0.00 & 5.00 \\
\hline Brotogeris juguaris & II & B. Intervenido/ lugares abiertos & 0.00 & 1.67 \\
\hline \multirow[t]{2}{*}{ Iguana iguana } & II & $\begin{array}{l}\text { B. no intervenido, b. poco } \\
\text { intervenido, b. intervenido/ } \\
\text { lugares abiertos, áreas }\end{array}$ & & \\
\hline & & urbanas o suburbanas & 0.83 & 0.00 \\
\hline Otus cooperi & II & B. Intervenido/ lugares abiertos & & 5.00 \\
\hline \multirow[t]{2}{*}{ Crotalus durissus } & III & $\begin{array}{l}\text { B. No intervenido, B. Poco } \\
\text { intervenido, b. intervenido/ }\end{array}$ & & \\
\hline & & lugares abiertos & 0.00 & 1.67 \\
\hline Potos flavus & III & B. Intervenido/ lugares abiertos & 1.67 & 0.00 \\
\hline
\end{tabular}

(Cordia dentata), Papalón (Coccoloba caracasana), Papaya (Carica papaya), Tempisque (Sideroxylon capiri subsp. tempisque), Guapinol (Hymenaea courbaril), Guacimo de molenillo (Luehea candida), Chocoyito (Diospyros salicifolia), algunas especies de las familias Moraceae, Euforbiaceae, Sapotaceae entre otras.

Cercar las áreas forestales y evitar el ingreso de animales domésticos o cazadores furtivos.
Repoblar con especies de fauna silvestres como Iguanas (Iguana iguana), Garrobos (Ctenosauria similis).

Desarrollar estudios en el área de dinámica de poblaciones de Monos Congos (Alouatta palliata).

Incluir a los dos grupos más grandes de mamíferos (Orden Rodentia y Orden Chiroptera) en las siguientes investigaciones. 


\section{REFERENCIAS BIBLIOGRÁFICAS}

Emmons, LH. 1999. Neotropical Rainforest Mammals. Editions 2da. ed. sig. University of Chicago press, ISBN. $307 \mathrm{p}$.

Ceballos, G; Galindo, C. 1984. Mamíferos silvestres de la cuenca de México. Instituto de ecología y museo de historia natural de la ciudad de México. Ediciones limusa, primera edición. D. F, México. 300p.

Guariguata, MR; Kattan, GH. Compiladores. 2002. Ecología y Conservación de Bosques Neotropicales. Asociación de Editoriales Universitarias de América Latina y el Caribe (EULAC); Agencia de Cooperación Técnica Alemana (GTZ). 689p.

Harcourt, CS; Sayer, JA. 1996. The Conservation atlas of tropical forest. The Americas. Simon \& Schuster. 206211.

Hurtado, L. 2000. Biodiversidad en Nicaragua, un estudio de país. Managua, Nicaragua. 89 p.

Kohler, G. 2003. Reptiles de Centro América. Offerbach; herpeton Verlog, ISBN. 367P.

Krebs, JC. 1985. Estudio de la Distribución y la Abundancia. Segunda edición. Harla, S. A. de C.V. México. 753 p.

MARENA. 1994. Proyecto Nandarola/ MARENA- DED, Plan de manejo de la cooperativa Pedro Joaquin Chamorro Cardenal. Proyecto de protección del bosque. Managua, Nicaragua. 35p.

Montenegro, M. 2007. Estado del Ambiente en Nicaragua. Managua, Nicaragua. 274 p.

Roldan, H. 2001. Recursos forestales y cambio en el uso de la tierra, Republica de Nicaragua. Santiago, Chile. 73p.

Ruiz, GA; Buitrago, F. 2003. Guía Ilustrada de la Herpetofauna de Nicaragua. Primera Edición. Managua, Nicaragua. ARAUCARIA-MARENA-AECI. 337P.

Stiles, GF; Skutch, A. 2007. Guía de aves de Costa Rica. 4ta. ed Trad. L. Roselli, illus. D. Garner. Instituto Nacional de Biodiversidad, Heredia, Costa Rica. 576 pp. 